\title{
Research on the joint fault location and cross impact analysis method of information communication network
}

\author{
Wenting ZHOU ${ }^{1, a}$, Xuemei $\mathrm{LI}^{2, \mathrm{~b}}$, Limin $\mathrm{CUI}^{1, \mathrm{c}}$, Hongfei XU ${ }^{2, \mathrm{~d}}$, Qingsu $\mathrm{HE}^{3, \mathrm{e}}$, Xin \\ $\mathrm{SHI}^{3, \mathrm{f}}$
}

\author{
${ }^{1}$ China State Grid Xinjiang Information and Telecommunication Company, Urumqi, 830000, China \\ ${ }^{2}$ China State Grid JiBei Information and Telecommunication Company, Beijing, 100000, China \\ ${ }^{3}$ China State Grid Electronic Commerce Company, Beijing, 100000, China \\ aemail: zhouwenting@xt.xj.sgcc.com.cn, bemail: li.xuemei@nc.sgcc.com.cn, ${ }^{\mathrm{c} e m a i l}$ \\ cuilimin@xt.xj.sgcc.com.cn, ${ }^{\mathrm{d} e m a i l}$ :xu.hongfei @nc.sgcc.com.cn, email: \\ heqingsu@sgitg.sgcc.com.cn, ${ }^{f}$ email: shixin@sgitg.sgcc.com.cn
}

\begin{abstract}
Keywords: The Information and Communication Network; Fault location; Cross-impact analysis; Alarm Correlation Analysis
\end{abstract}

\begin{abstract}
The operation monitoring and alarm disposition of Information networks and Communication networks rely on two systems. Different networks will cause different fault alarms when a fault occurs. However, the networks could only recognize its own fault and alarm respectively. They can't feed the alarm information to each other, so they can't find the location of the fault. This paper mainly researches on the joint fault location and cross-impact analysis method of information communication network. Based on information communication protocol principles and innovation of network failure mechanism, this paper invented a new method on detecting joint fault location and cross-impact analysis of information communication network. This paper mainly investigated on three aspects: protocol rule, model design and algorithm analysis. The aim of this paper is to help the information communication network find fault location, and predict the fault, so that it will improve the efficiency of fault handling and increase the cooperation between the two networks.
\end{abstract}

\section{Introduction}

Nowadays, operators in the State Grid Corporation of China need to rely on two sets of systems to achieve network fault location and analysis the fault. This is because the communication transmission network and information network work in different layers of network protocols, the data standards and the interface standards are not uniform, and alarm disposal methods and analytical methods are different. As a result, these two networks are running and monitoring in different communication management systems, information operation \& maintenance integrated supervision systems. If something wrong happens in system, it especially the fault in the transmission network, maintenance person who are responsible for the application level can't locate the source of the problem. They also couldn't analyze the impact of the transmission network fault.

When there is a wrong Literature [1] focused on mining and prediction model of alert association rules of the communication network. Such as the aspects of network topology and alarm attributes, etc. By doing the analysis of alarm, this paper identify the relationship between these two parts so that it could accurately determine the root causes of fault. Literature [2] aimed at the problem of the omission of communication network fault which may cause the power accident. It invented a credible arguments minimum loss of fault location algorithm based on the weighted probability bipartite graph model. Literature [3] proposed an efficient algorithm for alarm association rule mining of communication network based on weighted frequent pattern tree. It also compared this algorithm with existing weighted association rules mining algorithm. Using this new algorithm will save a lot of storage space and improve mining speed. Literature [4] created a method of electric power communication network fault location intelligence analysis so that it can quickly and 
accurately locate the fault. Literature [5-8] investigated on the network alarm correlation analysis which can detect fault location, diagnosis and prognosis the fault. Increase the efficiency of troubleshooting.

As discussed above, there are a lot of research on alarm correlation analysis and fault location method for a communication network in the electric power industry. However,

less research for the joint fault location of information and communication network, cross-impact model of information network and communication network. This paper is focused on the method of the joint fault location and fault cross-impact of Information Communication Network

\section{Outline}

The author thought it should divided into three parts to investigate the joint fault Location and cross-impact analysis of information communication network: failure mechanism, the unification of the data model of communication and information network, cross-impact analysis algorithm and unified monitoring standards of communication information network.

Conduct a study of unified data model and monitoring standard based on SG-CIM model [9]. It established a research system from the following aspects:

(1) Mechanism of fault effect between the information communication network;

(2) Make data model and monitor standards unified;

(3) The algorithm of joint fault location and the method of cross-impact analysis;

(4) Visual support tool

The logical relationship was shown in Fig.1.

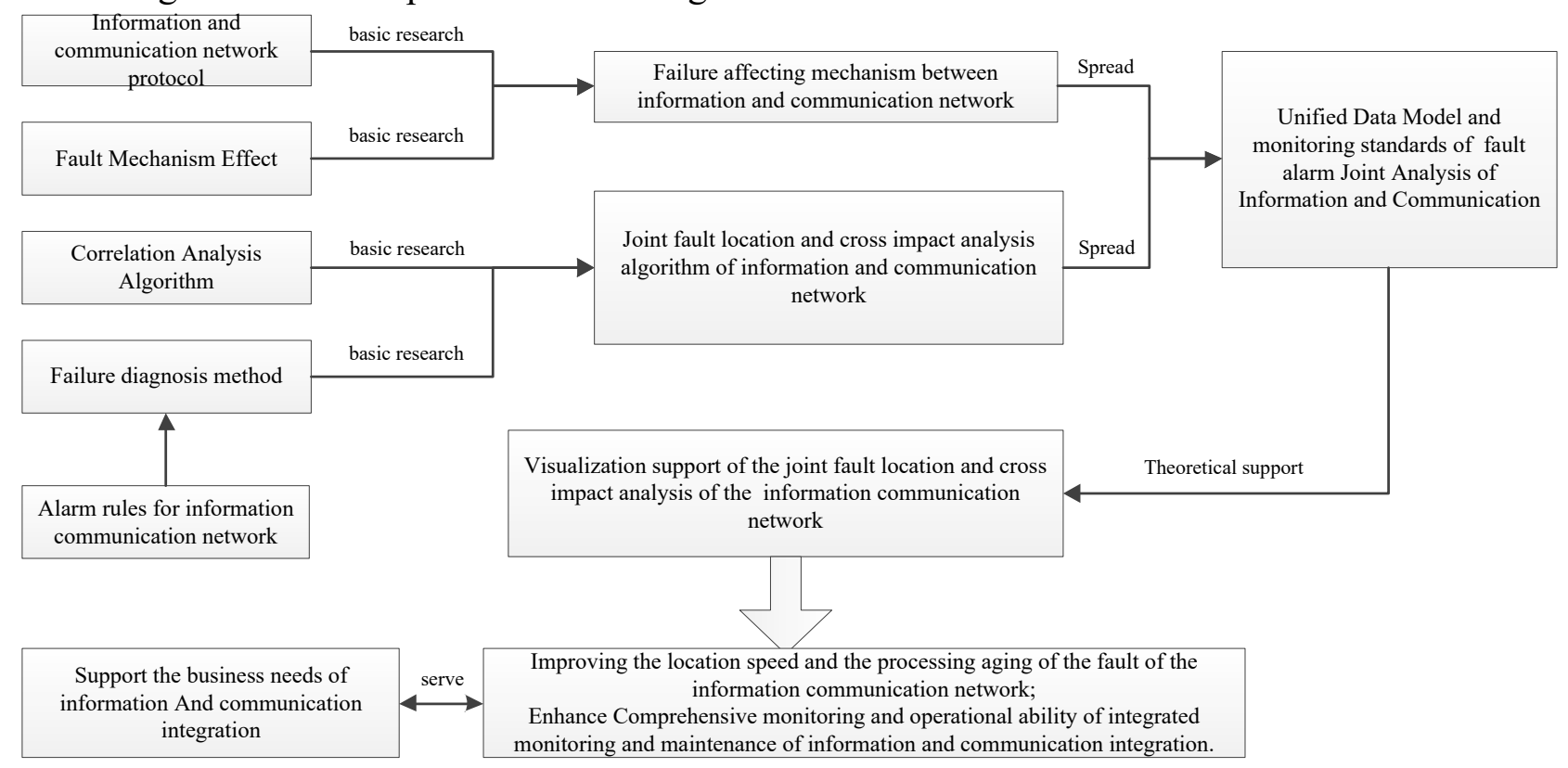

Fig1. Logical relationship

The current operation management information systems of the communication network and information network are independent of each other. Different network operation management information system has established equipment model and index model to meet the needs of self. Information network and communication network have been deployed to run. On the basis of this two networks, this paper proposed a research method of two network operation and maintenance management system interaction through algorithm analysis, data mining [10-13] and other technologies. The technology structure is shown in Fig2. :

To achieve the goal of joining cross location of information and communication network, it need to address the following questions first:

(1) existing data acquisition and standardization of two networks

When designed the existing operation management system, the data integration interface is not considered or weakened. This leads to the difficulty in data acquisition and data standardization. This can be solved by using SNMP, web service, file exchange, database exchange methods etc. to 
satisfy the acquisition requirements.

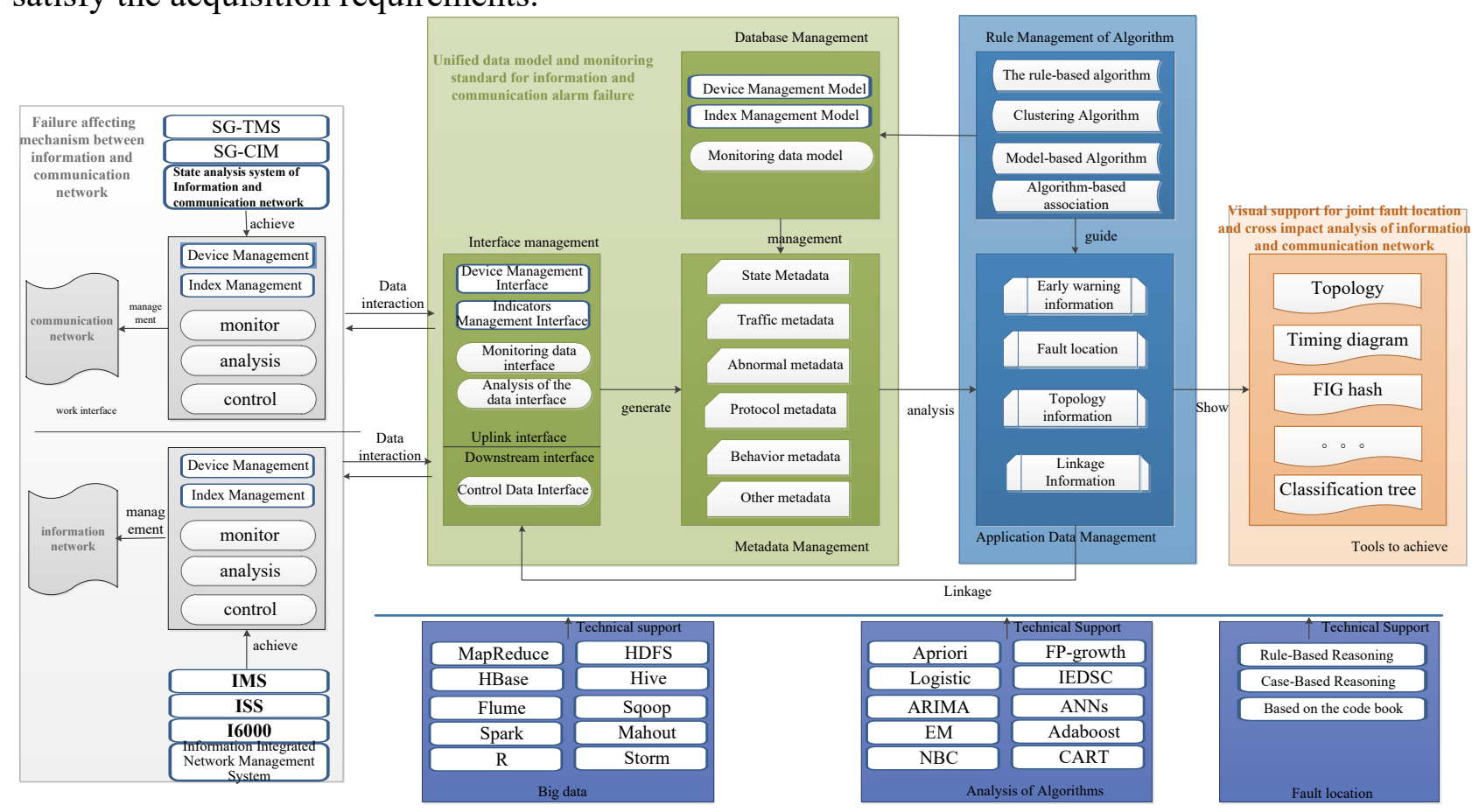

Fig2. Technical structure

Mechanism and Modeling of Fault Location Technology

It is still difficult to determine whether the specific algorithm meets the requirements of accuracy. The algorithm also has the stability test problem. This is because the technological systems of the two networks are different. The fault location analysis algorithm are quite different in their respective areas, and there are a lot of noise alarm data. The solution is to set multiple sets of management algorithm in parallel to detect network status continuously. Simulate the failure artificially to ensure a sufficient amount of sample data.

(2) Data processing delay and processing efficiency

The information of SGCC is large and doing history-related data analysis is extremely difficult.

Solution:

First, research on the algorithm selection, data acquisition, processing efficiency, selection of indicators and other aspects. Use big data to construct the efficient underlying platform for the data processing. Secondly, weigh the efficiency cost against availability cost effective. Finally, make the software designed to meet the basic principles which include safety, efficiency, function and etc.

(3) The influence of joint and cross-location to the existing system

The solution is to reduce the demand for data frequency and quantity by determining the scope of the method step by step.

\section{Fault influence mechanism between the Information Communication Network}

Through the analysis of the technology system, the network protocol and the working principle of information network and communication network, analyzing fault diagnosis algorithm, the alarm rules, and fault locating speed and processing time of the existing network of two networks. Combing the status, level and influence degree of the cross effect fault between network information network and communication network, establish the fault scenario model, to explore the failure mechanism of joint information communication network, formed fault influence mechanism model.

$>$ The technology system of information communication network

By Studying technology system of information network and communication network and doing research on monitoring technology of information communication network and its boundary region, this paper made the "translate" standard of operation and monitoring between the different technical system of information communication networks. This can do the joint fault root causes analysis and 
impact analysis of the cross information communication network.

$>$ The network protocol

Using the tools/techniques network protocols such as TCP/IP, SNMP, SYSLOG, Telnet, etc., network topology auto-discovery technology, network traffic analysis technology to provide technical support for network fault judgment analysis.

In recent years, UHV backbone grid and trans-regional power grid construction process is accelerated and company information construction have been developed comprehensively. The electric power information communication network becomes very large and complicated. As a result, various business of the national grid has been used which cover all levels of the grid sites, involving different manufacturers and types of equipment and transmission media.

$>$ working principle

Information network and communication network work in the different layers of network protocols. They adopt different technologies and standards. The communication network is used to carry power grid services network, works at the physical network layer and provide a wide area network access to the Internet to the information transmit channel. The information network is the outer ring of the communication network, works in network link layer, network layer, directly bears the operation business of communication and information. Communication network and information network achieve the protocol docking rely on DDF or photoelectric conversion module in the information network equipment. At present, this part of the "bridge" in the information network and communication network are not monitored. It belongs to surveillance blind. The premise of realizing the comprehensive centralized monitoring of the information communication network is to realize the real-time monitoring of the blind spot.

First, determine the strategy exist in the single network or between the two networks In the associated information communication network, namely network fault strategy or net fault strategy. The network fault strategy based on the relationship between the two networks is to define the connection or rely on associations, clarify the relationship and the influence of fault propagation. The network architecture of the causes of failure may be distributed in any one of these layers, classify network fault according to the five levels.

$>$ Fault diagnosis algorithm

Fault diagnosis and fault location are the core of the information communication network management. It is required to find the fault location and the reason in time so as to eliminate the fault and restore the system function when a fault occurs. One of the important means of fault diagnosis is correlation analysis technology, and it has been a hot spot in the field of expert research. In recent years, the experts of major areas are actively seeking the solutions of alarm correlation analysis intelligent. Such as China Telecom has set specifications of alarm correlation analysis system, and began to build intelligent alarm monitoring system.

Many methods are adopted in Alarm correlation analysis techniques, such as analysis of alarm correlation based on rules, analysis of alarm correlation based on examples ,analysis based on the relevance of the causal model, correlation analysis based on the model, correlation analysis based on neural network, etc. Foreign early have in-depth research in this area.

\section{Unified data model and monitor standard}

The important precondition to carry out the analysis and calculation is to establish the correct data model and realize the standard uniform data collection. Currently the real-time performance monitoring management of communication network and information network divide and rule,. It has not form the unified standards of monitoring standard, resource model, performance index, alarm rules did not form. It can't meet the requirements of "mutual understanding". Comprehensive analysis of Information Communication Network operation, studying the unified data model between "mutual understanding" fault alarm conjoint analyze of the information communication network based on SG-CIM modeling method, combing the distribution of two network alarm data. Research on the multi-stage network alarm correlation processing technology based on BP network. The network analysis model of fault data is established, and the fault diagnosis is made by using the 
analysis method of the bottom up the module. Exploring the monitoring mode of fault alarm joint analysis base on fault monitoring standard model of communication and information network operation and maintenance platform, completing the study on monitoring standard of fault joint analysis of information and communication network included the interface layer, data acquisition layer, data processing layer, data display layer four levels, a data source .

The construction steps of information communication network model:

(1) The establishment of power information communication resource management model

First, investigating and combing the electric power enterprise information communication resources, clarify the scope and depth of information communication resource management. Make manageable property of the information and communication resources clear. Analyzing the relationship between information and communication resources. Design the information communication resources management model suitable for electric power enterprise.

(2) Unify coding and modeling of information and communication repository

Base on the characteristic and classification of information communication resource in current electric power enterprise IT environment, referring to the relevant provisions of the electric power enterprise, unified coding for all kinds of information and communication resources of the whole company. Sum up the relationship between information and communication resource, establish a unified repository resource model.

(3) Establish a unified resource library of power information and communication

In view of the electric power business and establish business resource model: in-depth research o power systems and business use, establish business management model on the basis of a unified information and communication resource modeling, "combo" to integrate enterprise information resources, design and develop a unified information communication resource library system that provides data storage and maintenance service for electric power enterprise information communication management application.

Information communication network joint fault impact analysis of the main research contents are as follows: how to draw lessons from and deepen the information communication network fault impact analysis algorithm, build the information communication network joint fault effect analysis model, considering the network mode, logical link set, alarm derivative relations, business abstract analysis model of load mode (protection)

Specific studies include the following points:

- Combined with the characteristics of the demand and service support of power information communication network, mining the efficiency and accuracy of the SDH alarm root cause analysis algorithm.

Extract information network alarm determination model From the view of the business support and network analysis,, systematically standardized information network monitor indicators.

- Building joint failure analysis standardization alarm transmission model of information communication for the requirements of joint network fault analysis, by coding, description, resource identification, qualitative criteria, classification dimensions and other element of alarm, security alarm information consistent cognition in the joint analysis process of across the network.

- Construction of fault judgment index model of information communication network, concatenated fault judgment standard of heterogeneous information communication network, set algorithm auxiliary and experience analysis, assist to improve the efficiency with a fault in the operation and maintenance process.

In addition, in the process of network joint fault location model, still need to set out from the professional network itself, carry on their business model one by one, combine each professional network business model to become multi-level business view of relations with clear support, to ensure that more intuitive, logical relation clear analysis result transfer to operations staff.

\section{Joint fault location and cross-impact analysis algorithm}

Combing SGCC information integrated operational supervision system network alarm impact 
analysis, alarm correlation analysis function and the communication network management system of communication network alarm function of the root cause analysis. Using fault alarm analysis method, information networking protocol and fault influence mechanism model of two networks, studying the joint fault location [14] technology of information communication network. Overall planning information communications unified resource integration model and standard consistent with the monitoring data. Researching network between new joint positioning and cross-impact analysis algorithm and the locating diagnosis of fault for the influence of the two network. Analyze network QoS. Checking the network jitter that is difficult to locate before, the decrease of the quality of the network time delay, network, implement active operations and other network problems, further improve the operational quality and efficiency. Strengthen the two-way interaction between information communication network operation monitoring, realize two networks at the operational level of "vertical".

$>$ algorithm research

Analyzing the advantages and disadvantages of the existing data processing algorithm through studying the existing algorithms, for further study of the two network joint fault location and cross-impact analysis algorithm to lay a solid foundation.

Fuzzy c-means algorithm: implement the combination of the concept of clustering and fuzzy logic. Elements of fuzzy concentration are not belonging to a cluster, but belong to different clustering at the same time at different probability. FCM implementation is divided into $\mathrm{c}$ a $\mathrm{n}$ vector fuzzy cluster target, at the same time meets the targets in advance threshold value function requirements FCM algorithm has several characteristics, such sensitive to an isolated point, good adaptability to meet the needs of normal distribution data set.

D-S fusion theory: It is a theory of evidence and probability reasoning, it is mainly dealing with the problem of evidence weighted and evidence support, and use probability reasoning to achieve the combination of evidence, evidence theory is a kind of promotion of probability theory from the mathematical standpoint.

Apriori algorithm: It is one of the most influential algorithms for mining Boolean association rules frequent items etc. The core is a recursive algorithm based on the idea of the frequent item set in the two stage. The association rules on the classification are the unidimensional, single-layer, Boolean association rules. Apriori algorithm is widely used in various fields. Based on the data correlation analyze and mining, dig up information has important reference value in the process of decision-making. Two major drawbacks of Apriori algorithm are likely to produce a large number of candidate sets, and may need to repeat the scan database.

Analytic hierarchy process (AHP): APH is a kind of quantitative analysis of multiple criteria decision-making problem of qualitative methods This method is Simple, flexible and practical. Its characteristic is through the various factors of complex problems into interconnected orderly level, streamline, according to the structure of a certain objective reality of subjective judgment (mainly is the comparison of the two) the expert opinion and analysis of the objective judgment result directly and effectively combined with the importance of a two level elements compared to quantitative description. Then, by using the mathematical method to reflect each element order of relative importance weights, through all levels between the total sorts of calculating the relative weight of all the elements and sorting.

$>$ Crossover study analysis of the information communication network

With the continuous development of social information and more information integrated functional is required, the integration of computer network and communication system is an important direction for the future. Communication network and information network through the transmission system, routers and switches in the integrated data network.

Currently, when integrated data information network fault occurs, firstly you need trouble shooting exclusion timeshare segment to network transmission equipment, transmission link of communication, Secondly, judge the fault tolerance of information minicomputers, storage equipment, database, middleware, routers, switches, firewalls, load balancing and other business content related to the information on the level. In this way, the failure analysis time is too long. It is 
not conducive to the normal development of related businesses.

In order to improve the information communication network fault localization speed and processing time, improve the information communication comprehensive monitoring operational capacity after the fusion, we must strengthen the cross-impact analysis of the information communication network research. It is good for information communication network combined fault location.

\section{Research on visual support tools}

Combining with the visual representation with information data network management products performed data mining and visualization analysis to explore the multidimensional multi-scale evaluation mode of the data relationship. The study of the multi-level network topology fault autographic display technology for cross-boundary of the information communication network to realize the dynamic visualization view for the scope of fault impact and provide the function of graph model linkage and graphical show security alarm and system failures, provide the support tool of joint fault location and cross-impact analysis of information communication network, to service dispatching decisions.

Construct the comprehensive reference model and study the performance index system of main business systems. Rely on test data and professional network management monitoring data in-depth analysis of relationship and interaction in various parameters, establish alarm model, form a hierarchical classification evaluation standard system of the running status and performance of information communication systems and equipment, to provide technical verification support for maintenance and preventive maintenance.

\section{Prototype system application deployment}

Prototype system was deployed and applicated combined with the operation status of information communication network of SGCC, and the mode of "secondary deployment, the tertiary application" was adopted, and data was exchanged with SG-TMS and SG-IMS through the interface. The whole system application structure consists of headquarters (divisions), provincial system and the Internet.

The upper is composed of the headquarters (divisions) system, and the lower is composed of the provincial system. The upper system interconnect with each other through cross-regional information communication network to achieve the interconnection and information sharing of the cross-regional system and form the comprehensive management ability for cross-regional backbone of communication network and information network system. The upper and the lower layers of the system interconnect with each other through the network across the province to achieve the interconnection and information sharing of the system that across the province and forms the comprehensive management ability of the backbone communication network across the province. The lower system interconnect with each other through the province network to achieve interconnection and information sharing of the province system at all levels to form the comprehensive management ability of provincial backbone network.

\section{Conclusion}

The research method that solves the joint fault location and cross-impact analysis of information communication network is proposed in this paper, it completely expound the principle and process of the network fault location. Through the analysis of fault mechanism, monitoring standards, algorithms, building a prototype system, visualization tools showing and other aspects of the information communication network to define the feasibility of this research. It is helpful to improve the fault location efficiency and aging recovery of the information communication network and meet the requirement of the reliability and safety of the information communication network, it also can support the company to promote the integration of information and communication 
network effectively and accelerate the development of the information communication intensification.

The research method proposed fills the void of network joint and cross fault localization. The impact analysis of the fault location will be researched based on the mature technology of alarm management analysis and combined with the scene of the joint of information communication network.

\section{Acknowledgement}

In this paper, the research was sponsored by the Technology Project of SGCC ( Project name: The Research on the Key Technology of The Joint Fault Location and Cross Impact Analysis of Information and Communication Network).

\section{References}

[1] LI Tongyan. Researches on Data Mining Based Alarm Correlation Analysis in Communication Networks[D]. Ph.D. thesis. University of Electronic Science and Technology of China. 2010.

[2] WANG Kaixuan; YANG Zheng; QIU Xuesong. Algorithm of Fault Locating on Impact Analysis in Power Communications Network[J]. Journal of Beijing University of Posts and Telecommunications. 2014, S1.

[3] LI Tongyan, XIAO Hailin, and LI Xingming, Algorithm for Mining Weighted Alarm Association Rules in Telecommunication Networks[J]. Journal of University of Electronic Science and Technology of China., 2008,37(6): 807-810.

[4] WANG Xiang, DAI Fei, GAO Weizhong, et al. Fault Location for Electric Power Communication Network Based on Intersection Operation and Combined Fuzzy Condition[J]. Automation of Electric Power Systems, 2014,38(24):114-118.

[5] ZHAO Zhendong, HUANG Nan, LI Zihan. Alarm Correlation Analysis of Network Failure in SDH[J]. Telecommunications for Electric Power System, 2012,33(6): 63-66.

[6] Xiao Hailin, Li Xingming, Study of Analytic Hierarchy Process Applied to the Alarm Correlation Analysis in Communication Networks[J]. Telecommunications Science, 2006,22(11): 36-39.

[7] ZHANG Xianfei, HOU Sizu. Alarm correlation analysis for monitoring system in power communication network[J]. Telecommunications for Electric Power System, 2009,30(1): 47-50.

[8] Wang Yang, Li Guocai, Xu Yakun. Research on Management Method, Classification and Correlation of Alarm in Information Communication Network[J]. Telecommunications Science, 2013, 29(8) :132-135.

[9] LI Yisong, YANG Qi, HU Nan, et al. SG-CIM based Power Information System Monitoring Model. Electric Power Information Technology, 2012,10(10): 35-39.

[10]G. Grahne, J. Zhu. Fast Algorithms for Frequent Itemset Mining Using FP-Trees. IEEE Transactions on Knowledge and Data Engineering, 2005, 17(10): 1347-1382

[11]C. K.-S. Leung, Q. I. Khan, T. Hoque. CanTree: A Tree Structure for Efficient incremental Mining of Frequent Patterns. Proceedings of ICDM, Houston, Texas, USA, 2005, 274-281

[12]C. K. Leung, Q. I. Khan, T. Hoque. CanTree: a canonical-order tree for incremental frequent-pattern mining. Knowledge and Information Systems, 2007, 11(3): 287-311

[13]Q. Ding, Q. Ding, W. Perrizo. PARM - an efficient algorithm to mine association rules from spatial data. IEEE Transactions on Systems, Man, and Cybernetics, 2008, 38 (6): 1513-1524

[14]ZHU You-chan, DOU Bing-lin. Network fault location algorithm based on dependency graphs[J]. Journal of Dalian University of Technology, 2005, z1: 73-77. 